\title{
TRABAJO Y SALUD: EL ACCESO A LOS SERVICIOS DE SALUD DE LOS TRABAJADORES FORMALES EN LA ARGENTINA
}

\section{WORK AND HEALTH: ACCESS TO HEALTH CARE FORMAL WORKERS IN ARGENTINA}

\section{Maitena Fidalgo*}

\author{
RESUMEN
}

Este artículo buscar reflexionar sobre las formas de acceso a los servicios de salud de los trabajadores formales en la Argentina. La historia del sistema de protecciones sociales ha ubicado a la vinculación "trabajo formal-protecciones en salud" como eje referencial en la asignación y reconocimiento de derechos. Interesa analizar la transformación del sistema de protecciones sociales asociadas al trabajo que se redefinió a partir del proceso de reforma neoliberal de la década del 90 .

PALABRAS CLAVE: ARGENTINA * SALUD * TRABAJO * SERVICIOS DE SALUD * REFORMA NEOLIBERAL

\section{ABSTRACT}

This article seeks to reflect on the ways of access to health services of formal workers in Argentina. The history of the social protection system has been at the link "formal work-health protection" as reference axis in the allocation and recognition of rights. The interest is to do an analysis about the transformation of the social protection system associated with the work being redefined from the neoliberal reform process of the 90's.

KEYWORDS: ARGENTINA * HEALTH * WORK * HEALTH SERVICES * NEOLIBERAL REFORM

Grupo de Estudios sobre Políticas Sociales y Condiciones de Trabajo, Instituto Gino Germani, Universidad de Buenos Aires, Argentina (UBA) y además, Sección Consultorios Externos de la
División Servicio Social del Hospital General de Agudos Dr. I. Pirovano. Ministerio de Salud de la Ciudad de Buenos Aires, Argentina.

maitenafidalgo@gmail.com 


\section{INTRODUCCIÓN}

Las formas de acceso a los servicios de salud de los trabajadores formales en la Argentina, constituyen el tema central del presente artículo. La historia del sistema de protecciones sociales ha ubicado a la vinculación "trabajo formal-protecciones en salud" como eje referencial en la asignación y reconocimiento de derechos. Es justamente la transformación del sistema de protecciones sociales construido en el país ${ }^{1}$, a partir de la década del 90 , el contenido general en el cual se inscribe este artículo.

Los trabajadores formales en la Argentina han tenido un sistema de protecciones sociales en salud específico: aquel denominado de obras sociales. El sistema de salud argentino está conformado por tres subsectores: el público, el privado y el de la seguridad social. Este último es, como se dijo, el que define la forma de acceso y cobertura de los trabajadores formales a los servicios de salud. Dentro del subsector de la seguridad social encontramos a las obras sociales como entidades encargadas de la organización y gestión del seguro de salud de los trabajadores.

Lo interesante en este artículo es compartir algunas claves para pensar el proceso de reforma delineado en el campo de la salud en general y en la seguridad social en salud, en particular, porque los procesos de reforma neoliberal producidos en la década del 90 en el sistema de salud dan cuenta de una transformación social, política, económica y cultural sin precedentes $^{2}$. Como reflexionaba oportunamente Castel sobre la sociedad salarial, enfrentamos la transformación de una sociedad que se organizó por medio del trabajo asalariado y superó la oposición propietario-no propietario

$1 \quad$ Este artículo presenta resultados de la investigación que sustenta la realización del Doctorado en Ciencias Sociales de la UBA (tesis en elaboración) realizada en el marco del Grupo de Estudios sobre Políticas Sociales y Condiciones de Trabajo del Instituto de Investigación Gino Germani.

2 Se sugiere lectura de Grassi, Hintze y Neufeld (1994) ya que el mismo se constituye en un trabajo pionero sobre los procesos de reforma neoliberal en la Argentina. al "yuxtaponer a la propiedad privada otro tipo de propiedad, la propiedad social, de manera que pueda permanecer (el trabajador) fuera de la propiedad privada sin carecer de seguridad" (1997: 302). Así, la cobertura de las necesidades en el campo de la salud de los trabajadores se constituyó en un derecho de los mismos en la medida en que brinda protecciones y seguridades frente a las contingencias de la salud.

En el caso argentino, se explora la reformulación en la construcción de las seguridades en salud de los trabajadores, tomando en cuenta que ha sido a partir de la participación en el mercado de trabajo formal que se construyó el seguro social de salud. El proceso de reforma neoliberal producido en la década anteriormente referida, se constituye en un punto de inflexión para el análisis de las protecciones en salud en este país, por lo que se detendrá en las rupturas que el mismo produjo en el subsistema de las "obras sociales".

\section{LA SEGURIDAD SOCIAL EN SALUD: LAS OBRAS SOCIALES EN SU MOMENTO FUNDACIONAL (1940-1970). BREVE DESCRIPCIÓN}

La dimensión institucional-organizacional de la seguridad social en salud, ubica a las obras sociales como gestoras del seguro de salud de los asalariados formales argentinos.

Las mismas se basaron en la afiliación obligatoria a las entidades correspondientes a los distintos sectores de actividad laboral, organizadas bajo la órbita de los sindicatos del ramo. El esquema se basó, así, en el aporte compulsivo de un porcentaje del salario y en la contribución igualmente obligatoria de los empleadores, equivalente a un porcentaje sobre la nómina salarial. Es decir, la forma de financiamiento ha sido ( $y$ así continúa) compartida entre trabajadores y empleadores exclusivamente.

El origen de este Sistema de Obras Sociales se remonta a la década del 40 con la creación de una Comisión de Servicio Social (Decreto 30.655/44) encargada de brindar servicios sociales desde las empresas. Dicha norma puede ser considerada como:

... acto instituyente de la salud de los trabajadores como cuestión de estado, y 
ya no dependiente de la buena voluntad o disposición de los empleadores, pero no para que este asumiera la función como tal sino para que (...) lo reenviara a las organizaciones de trabajadores" (Danani, 2005: 284-285).

El espíritu de la propuesta era vincular la inserción laboral con la atención de la salud, aunque la medida tuvo alcances parciales $y$ limitado impacto.

Pero el acontecimiento más importante de afianzamiento y expansión del sistema de obras sociales sindicales ocurrió ya en 1970, cuando por la Ley 18610 se "generalizó el modelo, universalizó la cobertura al declararla obligatoria para todos los trabajadores en relación de dependencia y unificó los descuentos salariales y la responsabilidad de los empleadores" (Danani, 1994: 17). De allí se pueda afirmar que constituyó una medida que se inscribió en una política pública, la cual estableció los criterios de funcionamiento y generalizó la distribución de los costos de la atención de la salud de los trabajadores con los empleadores. Esta ley terminó de institucionalizar el subsector de las obras sociales, a la vez que lo reguló y normatizó, por tanto, en definitiva puede decirse que este subsector del sistema de salud fue fundado a partir de la obligatoriedad $y$ uniformidad de los aportes y contribuciones a la entidad sindical correspondiente a la actividad laboral. Estableció, asimismo, los criterios de solidaridad entre los trabajadores de cada sindicato-obra social, que recibían una misma cobertura con independencia del monto de los aportes y las contribuciones que realizaban.

Posteriormente, con las leyes 23660 (Ley de Obras Sociales) y 23661 (Sistema Nacional del Seguro de Salud) — ambas aprobadas en diciembre de 1988 y promulgadas en enero de 1989 - las obras sociales quedaron constituidas como un sistema de seguro médico de carácter obligatorio establecido para todos los trabajadores formales (relación de dependencia) $y$ sus familiares directos. Desde entonces, estas funcionan como entidades de derecho público no estatal, con individualidad jurídica, financiera $y$ administrativa, no tienen fines de lucro $y$ están sujetas a la regulación del Estado.
Esas normas, además, obligaron al trabajador a afiliarse a la obra social correspondiente a su rama de actividad laboral, como única opción, por lo tanto, a contar con los servicios y profesionales que cada entidad ofrecía. Cualquier alternativa diferente (otros servicios médicos públicos o privados) corría por fuera del subsector de la seguridad social, aunque se mantenía la obligatoriedad de continuar realizando los aportes.

En síntesis, lo que se ha mantenido vigente hasta la década de 1990, es la "afiliación obligatoria" a la obra social correspondiente a la actividad laboral y la administración de la misma por parte del sindicato correspondiente. Así, la denominada "cautividad" de los trabajadores a la obra social administrada por el sindicato de la rama de actividad ha sido un rasgo estructural del subsector de las obras sociales. Este es el escenario con el cual se inicia la década de 1990, que da pie a los fuertes cuestionamientos al sistema: el principal argumento para la crítica era la falta de competencia entre las entidades, lo que redundaba - se decía- en un servicio deficiente, caracterizado por la mala atención y la disconformidad de los usuarios. Argumentos como estos contribuían a impulsar la necesidad de transformaciones en el subsector.

\section{EL MOMENTO REFORMISTA: LA DÉCADA DEL 90 Y EL PARADIGMA NEOLIBERAL EN LA SALUD}

El escenario descripto es modificado a partir de la política denominada de "desregulación de las obras sociales". Las condiciones generales y las reglas de esta dimensión institucional-organizacional se redefinieron en la década del 90, a partir de la instauración del derecho de los trabajadores formales comprendidos en el Régimen Nacional de Obras Sociales (Ley 23660 y 23 661) de elegir el Agente del Seguro de Salud.

En el marco de las reformas neoliberales, la liberalización del mercado de trabajo y la flexibilización de relación laboral fueron ejes centrales de la transformación entonces encarada. Esto impactó en el sistema de salud debido a la histórica construcción de los derechos sociales asociados al trabajo asalariado formal. Cuando 
el empleo protegido, estable y por tiempo indeterminado perdió la centralidad que había sabido ganar, los derechos sociales se constriñeron al ritmo del crecimiento del desempleo y la precariedad laboral. La retórica neoliberal desencadena, como sostiene Alvarez Uría (1998), una "mercantilización cada vez más profunda del espacio social", impregnando de lógicas propias del mercado al conjunto social. El campo de la salud no fue ajeno a dicho proceso mercantilizador.

Las negociaciones y disputas políticas producidas a partir del espíritu reformista que existía a principios de los 90 , en este caso, dispararon las luchas entre los diferentes actores del campo de la seguridad social y la salud. Como en todo intento de reforma, en este caso también los intereses y las resistencias caracterizan el proceso de conformación de la política pública de una manera singular. No fueron los propios trabajadores los protagonistas de estas estrategias sino quienes defendían intereses corporativos, solo explicables a través del análisis de la historia argentina. Sindicatos y empresas de medicina prepaga ${ }^{3}$ argumentaban buscando su mejor posicionamiento en el escenario post reforma. Las protecciones de los trabajadores formales aparecían, en ese escenario, como simple objeto de disputa $y$ de interés corporativo.

Fue el Decreto Presidencial nro. 9 de 1993, el que marcó la clave de la transformación y sentó las bases institucionales que continúan vigentes hasta la actualidad. Por dicha norma se estableció que los beneficiarios de las obras sociales comprendidas en la Ley 23 660 tendrían libertad de opción de su entidad entre aquellas que conformaban el conjunto de "obras sociales sindicales". El argumento central para esta disposición era que la "libre elección de obra social" por parte de los trabajadores tornaría más eficiente al sistema por la competencia que establecía entre las entidades. Se abrió, así, una contienda por afiliados entre las entidades sindicales integrantes del subsector de la seguridad social en salud, en tanto las

3 Entidades comerciales que integran el subsector privado del sistema de salud argentino y que comercializan seguros privados de salud. empresas de medicina prepaga quedaron formalmente excluidas de la pugna.

La innovación de las condiciones del subsector de la seguridad social, desde $1993^{4}$, a partir de la posibilidad de libre elección de obra social al interior del Régimen Nacional de Obras Sociales (Ley 23660 y 23 661), redefinieron las reglas de juego de ese campo. Ahora cada trabajador puede elegir cambiar el destino de sus aportes de una entidad sindical a otra, rompiendo con la "cautividad" de la obra social de la actividad laboral. Se postula entonces la singularidad del proceso de reforma: la definición de una política de libre elección de obra social junto con la definición del aporte como "personal" de cada trabajador formal.

¿Cuál es la principal ruptura que se produjo? Ni más menos que la posibilidad de movilidad de los afiliados al interior del subsector de la seguridad social (entre las diversas obras sociales sindicales), lo que según la hipótesis, le asignó a los trabajadores - a sus prácticasun marcado poder instituyente en la configuración actual del subsistema, a la vez que aportan activamente a la construcción de nuevas formas de vinculación con las protecciones sociales.

\section{ALGUNAS CLAVES PARA ANALIZAR EL PROCESO DE REFORMA EN SALUD EN LA ARGENTINA}

La política de "desregulación de la seguridad social" transformó el escenario de la protección de la Seguridad Social en Salud, principalmente en virtud, entonces, de la denominada política de "libre elección de Obra Social". A la vez posibilitó el desarrollo de diferentes prácticas por parte de los trabajadores $y$ de las entidades, en la medida en que las condiciones y las reglas del subsector así lo permitían.

Se examinan, a continuación, dos núcleos de sentido, por considerar que condensan los rasgos fundamentales de la transformación, a la vez que permiten recuperar el carácter instituyente de las prácticas de los trabajadores formales en el nuevo escenario. Ellos

$4 \quad$ La política de libre elección recién se hizo efectiva por falta de reglamentación en 1998. 
son: a) la libre elección de obra social en condiciones de personalización del aporte como clave para la comprensión de la reforma $y$ b) a las empresas de medicina prepaga como testigos visibles y protagonistas invisibles del escenario de la seguridad social.

\section{A) LA LIBRE ELECCIÓN DE OBRA SOCIAL EN CONDICIONES DE PERSONALIZACIÓN DEL APORTE ES LA CLAVE DE COMPRENSIÓN DE LA REFORMA}

Los términos en los cuales se concretó la reforma de la Seguridad Social en Salud instauraron el mecanismo de la personalización del aporte en tanto individual y particular de cada trabajador como eje central de las condiciones generales del subsector de las obras sociales, haciendo que los trabajadores puedan definir una estrategia de cambio o permanencia en la obra social que deseen. Los afiliados no pueden elegir entre realizar o no el aporte correspondiente, pero sí pueden direccionar el mismo según sus intereses. La posibilidad de elegir la obra social se definió como un derecho de los trabajadores.

Además, esto lleva al reconocimiento de la capacidad instituyente de las prácticas de los trabajadores, situación que no era tal en condiciones de cautividad de los mismos en la obra social asignada por la actividad laboral. En esas condiciones de cautividad a la obra social correspondiente a la actividad laboral, los empleados aportaban en forma proporcional a sus sueldos pero recibían prestaciones uniformes con independencia del aporte. Ahora el aporte es definido como un seguro "personal", por lo que los aportes y contribuciones correspondientes depositados en la obra social de origen, son remitidos a la que es elegida por el trabajador cuando este decide mudar de entidad, no quedando ningún porcentaje en la obra social de origen.

Estas condiciones generaban la posibilidad de vaciamiento de las entidades o el desfinanciamiento de las mismas, por lo que las obras sociales debían ahora competir en la búsqueda de afiliados. En consecuencia y simultáneamente, los trabajadores formales de más altos ingresos resultaron más atractivos para las entidades sindicales que aquellos de bajos ingresos, precisamente por su capacidad de aporte ${ }^{5}$.

Ante este panorama, la estrategia de algunas de las obras sociales sindicales fue el establecimiento de convenios de atención con empresas de medicina prepaga. Esto se convirtió, a la vez, en un factor de atracción de afiliados que deseaban acceder a la medicina prepaga $y$ que en el escenario post reforma encontraron la forma de hacerlo, por medio de la Seguridad Social en salud y el consecuente "aprovechamiento económico" de sus aportes a la misma.

En el marco de dichos convenios, las obras sociales transfieren a la entidad privada la totalidad de la gestión del seguro de salud, pero cobran un porcentaje del aporte en concepto de gastos administrativos por la intermediación. Estos afiliados indirectos a la medicina prepaga mantienen, para el organismo gubernamental de control de las obras sociales (Superintendencia de Servicios de Salud), la afiliación a la entidad sindical, lo que motiva que no se cuente con datos oficiales sobre la población de trabajadores que se encuentran en esta situación.

\section{B) LAS EMPRESAS DE MEDICINA PREPAGA} COMO TESTIGOS VISIBLES Y PROTAGONISTAS INVISIBLES DEL ESCENARIO DE LA SEGURIDAD SOCIAL

Si bien, los diferentes proyectos de reforma neoliberal debatidos en nuestro sistema de salud conjeturaban sobre la posibilidad de que las condiciones de competencia entre las entidades sindicales incluyeran también a las empresas de medicina prepaga, las reglas de la política de la libre elección excluyeron formalmente a las entidades privadas. No obstante, como se analiza en Fidalgo (2008), las condiciones de la política examinada permitieron que las empresas encontraran un lugar en el nuevo escenario. El producto de dicha participación ha sido la figura de los asociados indirectos;

$5 \quad$ En relación con este tema podría ampliarse el análisis a los mecanismos de redistribución entre diferentes entidades $y$ trabajadores, especialmente en virtud del proceso de reforma neoliberal. Pero este tema excede el objetivo del artículo. 
es decir, aquellos trabajadores que acceden a la medicina prepaga, a través de la seguridad social. Han sido los convenios de atención establecidos entre las obras sociales sindicales $y$ las empresas de medicina prepaga una fuerza generadora de traspasos entre las entidades sindicales $y$ un motor de crecimiento de entidades que por su naturaleza institucional, tenían un "techo" de captación de afiliados (aquel marcado por el crecimiento de la actividad correspondiente).

El punto más interesante de los procesos de reforma radica en las formas de vinculación entre ambos tipos de entidades, por las cuales las obras sociales establecen convenios de atención con las empresas de medicina prepaga. Entonces, por dichos convenios, las entidades sindicales derivan la atención de sus afiliados a determinada empresa. Así, el afiliado puede "moverse" por el sistema de obras sociales con su aporte, eligiendo aquella entidad sindical que le brinda el acceso a determinada empresa de medicina prepaga. Por ello, se señala que las empresas de medicina prepaga son "protagonistas" de la configuración actual de la seguridad social en salud, aunque no con la visibilización institucional que asumen desde el subsector de la seguridad social. Es decir, que no fueron pasivas observadoras de la transformación del campo, sino que definieron articulaciones con el sindicalismo argentino que les permitió vincularse con las obras sociales sindicales $y$ aumentar la cantidad de personas (clientes) que acceden a su cobertura. Puede ocurrir que los asociados, según su nivel de ingresos y la composición del grupo familiar, tengan que realizar un aporte de "bolsillo", es decir, un pago efectivo de dinero para completar el monto de la cuota mensual que cobra la prepaga. Pero en definitiva, ahora los trabajadores formales, los cuales en tanto tienen derecho la cobertura de salud por una obra social, pueden elegir o no, cambiar de entidad, asimismo, por esta vía indirecta y según su capacidad de pago (niveles de ingreso) pueden tener acceso a la medicina privada. Es decir, hallaron así, una puerta de entrada a un espacio de pertenencia hasta entonces negado. Por otra parte, dado que algunas familias de sectores asalariados con ingresos que lo permitían, además de tener su obra social se habían adherido desde antes de estas normas, a un seguro privado de salud que pagaban privadamente. La reforma de la seguridad social en salud les representó la posibilidad de un ahorro, al poder derivar el aporte a la obra social para el pago de la entidad privada.

Esta nueva "vía de acceso" a la medicina privada es considerada relevante, tanto por su magnitud $^{6}$ como por la generación e instalación de lógicas propias de los seguros privados dentro del mundo de la seguridad social. Esto es viable por dos motivos que se alimentan mutuamente: la reforma institucional y las prácticas de los trabajadores.

Esta estrategia de cambio de obra social buscando acceder a la medicina prepaga, no se ofrece por igual a todos los trabajadores: los niveles de ingresos como también, el lugar de residencia se constituyen en elementos diferenciadores en la posibilidad de acceder a la atención de una empresa de medicina prepaga. Dicha eventualidad se concentró en los grandes centros urbanos, en los cuales la presencia de estas empresas es significativa dadas las características de desarrollo y expansión del mercado de la salud. El condicionamiento según los niveles de ingresos actúa, no en el caso de un trabajador que opte por cambiar de una entidad sindical a otra, sino en aquellos que evalúan la posibilidad de cambiar buscando adherirse, a través de la obra social elegida, a un seguro médico prepago. De allí, entonces, que el margen de libertad

$6 \quad$ Si se considera la información brindada por el organismo estatal de control de las obras sociales sindicales (Superintendencia de Servicios de Salud) referida a los traspasos, se observa que las tres obras sociales que encabezan el ranking, a mediados de 2011, son: Obra Social de Comisarios Navales (269 418 opciones), Obra Social del Personal del Automóvil club Argentino (262 683 opciones) y Obra Social del Personal del Organismo de Control Externo (257 773 opciones). Dichas obras sociales mantienen convenios de atención con las tres empresas de medicina prepaga que mayor presencia tienen en el campo de la Salud: OSDE (Organización de Servicios Directos Empresarios es una obra social del personal de dirección, pero a través de la figura del afiliado adherente funciona ofreciendo seguros privados de salud), GALENO $y$ SWISS MEDICAL, respectivamente. 
para "elegir" es más o menos restringido o más o menos amplio, según la capacidad de pago (niveles salariales) de los usuarios.

En síntesis, se afirma que el reemplazo del criterio de la cautividad de los trabajadores a la entidad sindical en lo referido a su seguro de salud, por la libertad de elección (aunque con las restricciones ya mencionadas) habilita el desarrollo de prácticas por parte de los sujetos que definen su estrategia: eligen, se abstienen, averiguan, se cambian, se arrepienten, comparan... $y$ en definitiva, construyen el sistema "desde abajo".

EPÍLOGO. ¿POR QUÉ AFIRMAMOS QUE LA INDIVIDUALIZACIÓN Y PRIVATIZACIÓN COMO PROCESOS SE IMPUSIERON EN EL CAMPO DE LAS PROTECCIONES SOCIALES?

Porque la política de libre elección de obra social representa la formalización de las condiciones por las cuales los trabajadores hacen uso de su aporte/contribución definido como "personal", en función de una estrategia individual/familiar sobre el acceso a los servicios de salud.

La alianza entre obras sociales y empresas de medicina prepaga habilita el desarrollo de estrategias en torno de la atención de la salud, que "fugan" la cuestión de la atención de la salud de los trabajadores desde el subsector de la seguridad social hacia el subsector privado en salud, o más específicamente, hacia un lugar intermedio ubicado en la intersección entre ambos. Pero que, en definitiva, le otorgan al sujeto la potestad de definir su estrategia de atención de su salud con relativa independencia de los mecanismos colectivos de representación $y$ financiamiento que caracterizaron al subsector de la seguridad social.

Beck (2003: 30) reflexiona sobre la noción de individualización en términos de "desinscrutar sin reinscrustar". Allí, radica la lógica de la transformación: una estrategia colectiva de protección establecida como obligatoria frente a los riesgos de la clase trabajadora (con las controversias que pueda generar) cedió su lugar a una lógica basada en la individualidad como principio de organización y financiamiento.
Los cambios en el mercado de trabajo y en la relación salarial, han sido parte medular de la transformación de la situación de las personas en relación con sus protecciones, involucrando desde el diseño institucional hasta las estrategias más particulares. No obstante, la posibilidad de afrontar los riesgos y las contingencias de la vida continúa dependiendo de la participación de los sujetos en el mercado de trabajo. En efecto, esa participación sigue definiendo la situación de las personas respecto a las protecciones, tanto si ellas son de carácter colectivo y están inscriptas en la relación laboral, como si se trata de estrategias individuales derivadas de la capacidad de pago. De allí que se planteara una privatización hacia la esfera individual/familiar del trabajador de la responsabilidad ante la contingencia de enfermar.

Como reflexiona Danani, en relación con la "individualización de la protección", la misma refiere a una:

... forma específica de la orientación de la protección que se caracteriza por atar las condiciones de la vida de las personas a la situación y las prácticas en sentido amplio del sujeto en tanto individuo $y$ que a la vez desvincula esas condiciones de vida y las consecuencias de esas prácticas de toda relación con $y$ de toda pertenencia a grupos o agregados mayores (Danani, 2005).

En la seguridad social en salud, se redefinió la forma de construcción del esquema de protecciones en la medida en que se introdujo un criterio de individualización en un esquema como el de las obras sociales, definido inicialmente por el hecho de que era en función de la pertenencia a cierto grupo de trabajadores que se accedía a ciertos derechos. Dicha transformación solo puede pensarse $y$ analizarse considerando la mutación del "trabajo" en la sociedad capitalista y en las múltiples aristas que involucra: tanto como medio para la reproducción, como en su consideración de constructor de identidad.

Se entiende que el sistema de protecciones sociales definido a partir del trabajo, estableció un sistema de derechos que "protegió" 
las formas de acceso de los trabajadores a los servicios de salud, dando cuenta de un diseño institucional basado en el seguro social. Su transformación, además, permitió el ingreso de lógicas propias de los seguros privados de salud en el subsector de la seguridad social. En definitiva, se afirma que lo ocurrido fue una privatización de la responsabilidad por la salud de los trabajadores, siendo la capacidad de pago y/o el nivel de salario de cada empleado y su grupo familiar, el criterio que pareciera definir las estrategias posibles. Pero "estar protegido", como recuerda Castel (2004: 21) no corresponde a un estado "natural" sino que se trata de una construcción, que necesariamente remite a las formas de vinculación de los sujetos con la sociedad. En este caso, el avance en la libertad (relativa) de cada uno se produjo sobre la solidaridad de los grupos implicados.

BIBLIOGRAFÍA

LIBROS

Beck, Ulrich y Beck-Gernsheim, Elisabeth. La individualización. El individualismo institucionalizado y sus consecuencias sociales y politicas. Buenos Aires: Paidós, 2003.

Castel, Robert. La metamorfosis de la cuestión social. Buenos Aires: Paidós, 1997.

Castel, Robert. La inseguridad social. ¿Qué es estar protegido? Buenos Aires: Manantial, 2004.

Fidalgo, Maitena. Adiós al derecho a la salud. El desarrollo de la medicina prepaga. Buenos Aires: Editorial Espacio, 2008.

Fidalgo, Maitena. "Riesgos y contingencias de la vida: estrategias e institucionalidad confusa". El mundo del trabajo y los caminos de la vida. Danani, Claudia y Grassi, Estela (orgs.). Buenos Aires: Espacio Editorial, 2009.
Grassi, Estela y Danani, Claudia (orgs.). El mundo del trabajo y los caminos de la vida. Buenos Aires: Espacio Editorial, 2009.

Grassi, Estela; Hintze, Susana y Neufeuld, M. Rosa. Politicas sociales: crisis y ajuste estructural. Buenos Aires: Espacio Editorial, 1994.

\section{REVISTAS}

Álvarez Uría, Fernando. "Retórica neoliberal. La gran ofensiva de los científicos sociales contra las políticas sociales en EEUU". Revista Claves de Razón Práctica. Madrid: Ediciones PROGRESA (Promotora General de Revistas), 1998.

Danani, Claudia. "El Proyecto de Libre Afiliación a las Obras Sociales: análisis y perspectivas". PRONATASS: Planificación y Gestión de Politicas Sociales. Buenos Aires, 1994.

TESIS

Danani, Claudia. "La constitución sociopolítica de la relación asalariada: obras sociales y sindicatos en la Argentina, 1960 2000". [Tesis de Doctorado en Ciencias Sociales]. Universidad de Buenos Aires, 2005.

Fidalgo, Maitena. "Acerca del seguro privado de salud: reconstrucción histórica y configuración actual". [Tesis de Maestría en Políticas Sociales]. Universidad de Buenos Aires, 2007.

Fecha de ingreso: 29/11/2011 Fecha de aprobación: 10/02/2012 